\title{
Brachial Plexus Neuropraxia after CT-Guided Radiofrequency Ablation in a Patient with a Narrow Costoclavicular Space: A Case Report
}

\author{
John Paul Lew, Yan Ru Tan, Yee Yian Ong \\ Division of Anaesthesiology, Singapore General Hospital, Singapore \\ Email: John.paul.lew.h.j@singhealth.com.sg
}

How to cite this paper: Lew, J.P., Tan, Y.R. and Ong, Y.Y. (2017) Brachial Plexus Neuropraxia after CT-Guided Radiofrequency Ablation in a Patient with a Narrow Costoclavicular Space: A Case Report. Open Journal of Anesthesiology, 7, 357-363. https://doi.org/10.4236/ojanes.2017.711036

Received: August 10, 2017

Accepted: November 14, 2017

Published: November 17, 2017

Copyright ( $) 2017$ by authors and Scientific Research Publishing Inc. This work is licensed under the Creative Commons Attribution International License (CC BY 4.0).

http://creativecommons.org/licenses/by/4.0/

\begin{abstract}
A case of brachial plexus neuropraxia occurring during CT-guided radiofrequency ablation is reported in a patient with a narrow costoclavicular space. We discuss clinical methods of identifying patients with a narrow costoclavicular space who are at risk of position-related injury of the brachial plexus during anaesthesia. Identifying high risk patients and proper positioning during anaesthesia are hallmarks of preventing such injuries.
\end{abstract}

\section{Keywords}

Brachial Plexus Injuries, Anaesthesia, Interventional Radiology, Narrow Costoclavicular Space, Complication

\section{Introduction}

Brachial plexus neuropraxia during anaesthesia has been reported in multiple different surgeries and is caused by the stretching and compression of the plexus secondary to positioning [1]. Anatomical abnormalities of the thoracic outlet with which the plexus runs through as a contributory aggravating cause of such injuries have however been less discussed.

We describe a case of brachial plexus neuropraxia after radiofrequency ablation in a patient with a previously unknown narrow costoclavicular space and discuss ways of examining and identifying patients with thoracic outlet narrowing, which can increase their risk of postoperative neuropraxia.

The patient provided verbal informed consent for print and electronic publication of this case report. 


\section{Case Report}

The patient is a 54-year-old gentleman, $169 \mathrm{~cm} 62 \mathrm{~kg}$, with a history of diabetes mellitus, hypertension, hepatitis C complicated with Child's A liver cirrhosis on Peginterferon alfa-2a and ribavirin. He was diagnosed with primary hepatocellular carcinoma in hepatic segment 6/7. The lesion measured $3.1 \times 2.6 \times 2.7 \mathrm{~cm}$ and was indenting the retro hepatic inferior vena cava. He was scheduled for CT-guided radiofrequency ablation of the lesion.

The procedure was done in half left lateral position (body tilted 15 degrees to the left) on a CT table with both arms raised above his head i.e. elbows flexed, shoulders abducted and externally rotated, wrists pronated, and head in a neutral position. This position allows the proceduralist access and avoids CT image degradation from streak artifacts. All pressure points were protected during the procedure. The procedure was done under deep sedation for the first 1.5 hours; with a target controlled infusion of propofol titrated to sedation score. General anaesthesia had to be instituted for the last hour of the procedure as the patient was feeling uncomfortable and found it difficult to cooperate with the proceduralist. General anaesthesia was conducted with a supraglottic airway, spontaneous ventilation via a total intravenous anaesthesia technique with propofol. The patient remained haemodynamically stable throughout the procedure with no episodes of hypoxia. The entire duration of the procedure was 2.5 hours.

A few hours after the procedure, the patient complained about right upper limb weakness and numbness affecting mainly the $\mathrm{C} 5$ and $\mathrm{C} 6$ root distributions. Power (using the Oxford Scale) on shoulder abduction (C5) was 2/5, elbow flexion (C6) was $2 / 5$, elbow extension (C7) was $3 / 5$ and wrist extension (C6) $3+/ 5$. Sensory loss to pin prick was noted over the C5-C7 dermatomes. CT brain to rule out a stroke was normal. MRI brachial plexus done the next day showed abnormal enhancement (Figure 1) along the right brachial plexus involving the

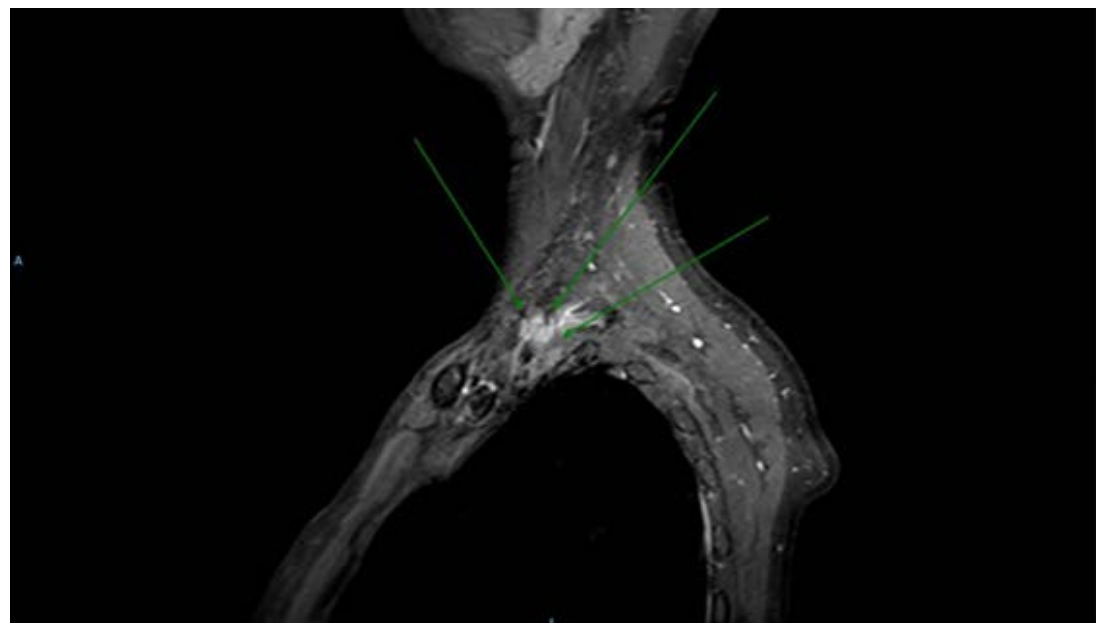

Figure 1. MRI right brachial plexus. The post-operative MRI right brachial plexus showed abnormal enhancement along the right brachial plexus involving the divisions and trunks segments suggestive of injury. The costoclavicular space was also noted to be narrow. Green arrows demonstrate the plexus enhancement. 
divisions and trunks segments from the costoclavicular space to the interscalene triangle. His costoclavicular space was noted to be narrow. The patient was given an arm sling and sessions with an occupational therapist for 1 month. He was also followed up by a neurologist. Nerve conduction studies done 1 month later were inconclusive for right brachial plexopathy. Fortunately for the patient, he recovered $90 \%$ of his right upper limb sensation and strength 3 months after the injury. Power on shoulder abduction was $4+/ 5$, elbow flexion was $4+/ 5$, elbow extension was $5 / 5$ and wrist extension $4+/ 5$. He had full recovery in motor strength after a period of 6 months.

\section{Discussion}

\subsection{Anatomy of the Thoracic Outlet}

The thoracic outlet is divided by the first rib into two sections. The proximal section of this canal is comprised of the interscalene triangle and the costoclavicular space, whereas the axilla comprised the distal section. Compression and stretching of the brachial plexus tends to occur in the proximal section of the canal. The interscalene triangle is bordered by the anterior scalene muscle anteriorly, the middle scalene muscle posteriorly, and the medial surface of the first rib inferiorly. The costoclavicular space is bordered anteriorly by the middle third of the clavicle, posteromedially by the first rib, and posterolaterally by the upper border of the scapula.

Both bony and soft tissue abnormalities can compress this tight space. Bony abnormalities include cervical ribs, an abnormal clavicle or first rib through exostosis, tumour, callus and fracture. Soft tissue abnormalities include congenital abnormalities of the scalene muscles and fibrous bands. These abnormalities can reduce the canal compliance and compress, stretch or kink the neurovascular bundle against the surrounding borders [2] [3].

\subsection{Anatomy of the Brachial Plexus}

The brachial plexus supplies all the cutaneous innervation of the upper limb except for the axilla and the dorsal scapula area. It also supplies all the motor innervation of the muscles of the upper limb except the trapezius and levator scapula.

The plexus is formed by the anterior rami of the cervical spinal nerves C5, C6, C7 and C8 and the first thoracic spinal nerve, T1. At the base of the neck, the roots of the plexus converge, forming three trunks, superior, middle and inferior. Within the posterior triangle of the neck, each trunk divides into two branches, an anterior and a posterior division. When entering the axilla they combine into three cords, lateral, posterior and medial. In the axilla and the proximal part of the arm, the three cords will split to five branches: radial, median, ulnar, musculocutaneous and axillary.

\subsection{Mechanism of Injury}

The plexus lies relatively superficial during its course down the neck and into the 
axilla. It also has 2 fixation points, the vertebra and prevertebral fascia proximally and the axillary fascia distally, thus making it vulnerable to injury from compression, stretching and kinking [4] [5].

Jackson and Keats in their cadaveric studies identified 5 positions that place undue tension on the plexus [6]:

1) Extension with lateral flexion of the neck to the contralateral side;

2) Abduction of the arm at more than 90 degrees to the body especially if the arm is externally rotated, as in our case;

3) Compression over the acromion;

4) Lateral pressure of the shoulder directly compressing the plexus by the humeral head;

5) Pulling the humeral head downwards directly onto the plexus.

Stretching of the brachial plexus results in minute intraneural capillary rupture with formation of small hematomas, thus resulting in compression and thus ischemia of the nerve bundles [1] [7] [8].

Nerve injuries can be classified based on the nerve pathology according to Seddon's classification [9]. Of interest to anaesthesia; neurapraxia occurs when there is damaged myelin with an intact axon. Recovery usually occurs in weeks to months and the prognosis is generally good. Axonotmesis occurs when there is axonal disruption but the endoneurium is preserved. The recovery from axonotmesis is variable. In neurotmesis, the nerve is completely transected with complete destruction of the connective tissue support. The prognosis is generally poor [8].

\subsection{Other Factors}

Apart from anatomical and positional factors, there are also multiple patient and surgical factors associated with peripheral nerve injury occurring with anaesthesia. These include intraoperative hypotension, hypovolemia, hypothermia, pre-existing neuropathy including vitamin B12 deficiency, diabetes mellitus, alcoholism and smoking. Surgical factors include long operative time and type of surgery, especially those where there is possible direct injury to nerves [1] [5] [10] [11].

\subsection{Prevention}

\subsubsection{Identifying Patients with a Narrow Thoracic Outlet}

Patients Can be identified via a through history and physical examination [2].

Patients with a narrow thoracic outlet can have symptoms and signs of vascular or neuropathic compromise. Symptoms related to an arterial thoracic outlet syndrome include pallor, coldness and cold intolerance and pain in the hand. When there is venous compression, patients can complain of pain after excessive activity, edema of the arm and paresthesia in the fingers and hand. Neurogenic symptoms include pain, paresthesia, numbness and weakness. They can also have Raynaud's phenomenon and cold intolerance. Our patient did not complain of any of these symptoms prior to the procedure. 
Provocative clinical testing for thoracic outlet syndrome has been reported to display high rates of false positive but are relatively simple to do and non-invasive.

The elevated arm stress test examines the result of loading the plexus throughout the thoracic outlet syndrome container. The patient is seated with arms above 90 degrees of abduction and full external rotation with head in neutral position. Patient then opens and closes hands into fists while holding the elevated position for 3 minutes. A positive test is where pain and paresthesia results in discontinuation of the test for relief of the pain.

The costoclavicular maneuver evaluates provocation produced by costoclavicular space narrowing. The patient sits straight with arms at the side. The radial pulse is then felt. The patient then retracts and depresses shoulders while protruding chest. The position is held for 1 minute. A positive test is where there is a change in the radial pulse or pain/paresthesia production.

Upper limb tension testing is sensitive for irritation of the neural tissue including cervical roots, brachial plexus and peripheral nerves. With the patient supine, the examiner depresses the shoulder girdle and abducts the shoulder to 110 degrees while applying slight extension and elbow flexion to 90 degrees. The forearm is then maximally supinated and the wrist and fingers extended. Finally, elbow extension is applied. The neck is laterally flexed to the contralateral side. This test is positive if symptoms are reproduced.

Extra caution should be taken if thoracic outlet narrowing is suspected.

Our patient had a narrow costoclavicular space, but this was not recognized prior to the procedure. Provocative clinical testing could have been done for him prior, and might have identified his condition, so that more care could have been taken to prevent the injury. Provocative clinical testing should be routinely done for all patients undergoing procedures in which brachial plexus injuries are possible due to positioning, as described by Jackson and Keats [6].

\subsubsection{Anaesthesia and Positioning}

General anaesthesia, as used in our case, as well as the use of muscle relaxants increase the risk of brachial plexus neuropraxia [7] as they prevent the patient from correcting non-physiological positions. If a sedation technique is used, the patient must be instructed to inform the operator if he should have any discomfort in his arm.

Avoiding an arm above head position is useful in reducing stretch on the brachial plexus. For CT-guided ablation, Shankar et al. [7] recommends positioning the arms crossed across the chest and slightly raised with padding from the chest. This succeeds in giving the operator access and also good CT images of the liver while reducing risk of brachial plexus neuropraxia. If an arm across chest position is not possible, frequent arm position changes and restricting the arm above head position for only vital portions of the procedure will also help. After this incident, our department now routinely avoids the arm above head position and position our patient's undergoing radiofrequency ablation as described by Shakar et al., this is especially so if the case is to be done under a gen- 
eral anaesthetic.

Intraoperative monitoring with somatic sensory evoked potentials have also been suggested and might be helpful [8] [12].

\subsection{Management of Brachial Plexus Neuropraxia and Prognosis}

In a patient suspected with brachial plexus neuropraxia, a thorough neurological examination should be done. Postoperative paresthesia, pain, numbness and weakness should be treated seriously and promptly. All motor or sensory loss should be documented. There might be a need to exclude stroke or a cervical spine injury especially if there are accompanying lower limb neurological signs. Management of the injury should involve a multidisciplinary team of neurologist, occupational therapist and physiotherapist. A magnetic resonance imaging of the brachial plexus can be done. Nerve conduction studies and electromyography (EMG) [5] may assist in diagnosing brachial plexus neuropraxia, as well as determining its age and severity. Typical EMG denervation changes in the upper limb appear 2 - 3 weeks after the injury. Early EMG helps to investigate for a pre-existing neuropathy.

Treatment of brachial plexus neuropraxia consists of mainly occupational therapy and physiotherapy. Arm exercises and muscle training can assist in its recovery by keeping the joints supple. Pain is controlled by simple analgesics and antiepileptic agents like gabapentin have been used for related neuropathic pain [8] [13].

The prognosis of brachial plexus neuropraxia is generally good with most recovering fully by 20 weeks. Ben-David B studied 22 patients with anaesthesia related brachial plexus neuropraxia after heart surgery. There was complete recovery in $68 \%$ of patients at a median follow-up of 20 weeks. Patients with diabetes had a higher risk of incomplete recovery [14].

\section{Conclusion}

Brachial plexus neuropraxia during anaesthesia is a thankfully relatively uncommon complication. It is caused by pressure or stretch on the plexus secondary to positioning. When it occurs, it may cause severe debilitation. A narrow costoclavicular space is one of the risk factors for brachial plexus neuropraxia. Screening for this condition and meticulous attention to positioning can help reduce the risk of neuropraxia. Provocative clinical testing, including the elevated arm stress test, the costoclavicular maneuver and upper limb tension testing, can be done to screen for thoracic outlet syndrome. Avoiding a general anaesthesia and an arm above head position can also help reduce the risk of brachial plexus neuropraxia.

\section{References}

[1] Cooper, D.E., Jenkins, R.S., Bready, L. and Rockwood, C.A. (2001) The Prevention of Injuries of the Brachial Plexus Secondary to Malposition of the Patient during Surgery. Clinical Orthopaedic and Related Research, 228, 33-41. 
[2] Hooper, T.L., Denton, J., McGalliard, M.K., Brismée, J.M. and Sizer Jr., P.S. (2010) Thoracic Outlet Syndrome: A Controversial Clinical Condition. Part 1: Anatomy, and Clinical Examination/Diagnosis. Journal of Manual and Manipulative Therapy, 18, 74-83. https://doi.org/10.1179/106698110X12640740712734

[3] Urschel, H.C. and Kourlis, H. (2007) Thoracic Outlet Syndrome: A 50-Year Experience at Baylor University Medical Center. Proceedings (Baylor University. Medical Center), 20, 125-135.

[4] Gagnon, J. and Poulin, E.C. (1993) Beware of the Trendelenburg Position during Prolonged Laparoscopic Procedures. Canadian Journal of Surgery, 36, 505-506.

[5] Shveiky, D., Aseff, J.N. and Iglesia, C.B. (2010) Brachial Plexus Injury after Laparoscopic and Robotic Surgery. Journal of Minimally Invasive Gynecology, 7, 414-420. https://doi.org/10.1016/j.jmig.2010.02.010

[6] Jackson, L and Keats, A.S. (1965) Mechanism of Brachial Plexus Palsy Following Anesthesia. Anesthesiology, 26, 190-194. https://doi.org/10.1097/00000542-196503000-00009

[7] Shankar, S., Vansonnenberg, E., Silverman, S.G., Tuncali, K., Flanagan Jr, H.L. and Whang, E.E. (2005) Brachial Plexus Injury from CT-Guided RF Ablation under General Anesthesia. Cardio Vascular and Interventional Radiology, 28, 646-648. https://doi.org/10.1007/s00270-004-0282-3

[8] Thomas, J. (2014) Post-Operative Brachial Plexus Neuropraxia: A Less Recognised Complication of Combined Plastic and Laparoscopic Surgeries. Indian Journal of Plastic Surgery, 47, 460-464.

[9] Seddon, H.J. (1943) Three Types of Nerve Injury. Brain, 66, 237-288. https://doi.org/10.1093/brain/66.4.237

[10] Parks, B.J. (1973) Postoperative Peripheral Neuropathies. Surgery, 74, 348-357.

[11] Warner, M.A., Warner, D.O., Harper, C.M., Schroeder, D.R. and Maxson, P.M. (2000) Lower Extremity Neuropathies Associated with Lithotomy Positions. Anesthesiology, 93, 938-942. https://doi.org/10.1097/00000542-200010000-00010

[12] Luginbuhl, A., Schwartz, D.M., Sestokas, A.K., Cognetti, D. and Pribitkin, E. (2012) Detection of Evolving Injury to the Brachial Plexus during Transaxillary Robotic Thyroidectomy. Laryngoscope, 122, 110-115. https://doi.org/10.1002/lary.22429

[13] Rose, M.A. and, Kam, P.C. (2002) Gabapentin: Pharmacology and Its Use in Pain Management. Anaesthesia, 57, 451-462. https://doi.org/10.1046/j.0003-2409.2001.02399.x

[14] Ben-David, B. and Stahl, S. (1997) Prognosis of Intraoperative Brachial Plexus Injury: A Review of 22 Cases. British Journal of Anaesthesia, 79, 440-445. https://doi.org/10.1093/bja/79.4.440 